\title{
Earth Science Needs Thorough Introspective Analysis and Renovation
}

\author{
Subhasis Sen* \\ Retired Scientist, Council of Scientific and Industrial Research, Kolkata-700094, India
}

${ }^{\star}$ Corresponding author: Subhasis Sen, Retired Scientist, Council of Scientific and Industrial Research, O-26, Patuli, Kolkata-700094, India; E-mail: ssennagpur82@yahoo.com

Received: August 04, 2021; Accepted: August 10, 2021; Published: August 28, 2021

\begin{abstract}
Despite several breakthrough discoveries, earth science needs modification especially related to its deeper parts of the planet where temperature and pressure have been presumed to be high. Based on the concept of earth's expansion, the author considers that the mantle of the unexpanded oceanless earth was considerably fluid owing to incorporation of ocean-forming water. In such a globe gravitational pull from an extraterrestrial planetary body would cause expansion triggered by swelling up of the semi-fluid mantle and consequent formation of a number of expansion cracks on the crust. Through these cracks or mid-oceanic ridges extensive molten magma would expel out and deposit on both sides of the crack to form ocean basins. Matching thickness of the earth's fluid outer core with the extent of expansion points out that owing to massive expansion original core-mantle conjunction of the planet was ruptured along which a void zone, identified as outer core of the prevalent concept, was gradually opened up. Such disposition of occurrence of two discrete geospheres separated by a virtually void zone would give rise to the phenomenon of reverse gravity in the deep interior of the planet thereby sustaining a low pressure and low temperature zone at depth.
\end{abstract}

\section{Introduction}

The author of this article, who is a geologist by love, education and commitment, while studying various features of earth science noticed that certain aspects of the studies call for introspective analysis and revamping and in a few cases rejection. Authur's study was conducted covering right from the crustal layer of the planet to its deepest part, including the enigmatic outer core. When the first authentic map of the globe was prepared in 1570 by Abraham Ortilius [1], which conspicuously exhibited parallel shore lines between Africa and South America. The pioneer cartographer not only noted this remarkable feature, but also for the first-time proposed occurrence of continents in the past in a conjoined manner. After that several observers, such as Antonio Snider-Pellegrini [2] of France as well as Alfred Wegener [3] of Germany and several other geologists attempted for continental adjustment. However, all such models were found to be untenable from the point of strict scientific study as they had liberally distorted the boundary of the relevant continents. I. O. Yarkovskii [4] of Russia for the first-time suggested Earth's expansion in 1888 while after that many scientists, some of them independently, conceived the idea of expansion of the Earth. In contrast to the adjustment done in an unaltered dimension of the earth, Hilgenberg [5] of Germany in 1933 showed that in a model of earth reduced to two/third of its original dimension perfect adjustment of continents could be achieved (Figure 1). This information evidently supports earth expansion theory to be a genuine concept suitable for explaining major global phenomena, while, for explaining such phenomena in a globe of unchanged dimension throughout the past geological ages requires several fabricated assumptions.

\section{All the World Loves a Fairytale}

In "The Problems of Philosophy" Bertrand Russell [6] expressed:

"Is there any knowledge in the world which is so certain that no reasonable man could doubt it? When we have realized the obstacles in the way for a straightforward and confident answer, we shall be well launched on the study of philosophy - for philosophy is merely the attempt to answer such ultimate questions, not carelessly and dogmatically as we do in ordinary life and even in the sciences, but critically after exploring all that makes such questions puzzling, and after realizing all the vagueness and confusion that underlies our ordinary ideas". Regarding apparently unquestionable notions, Sir Bertrand further pointed out that "Yet, all these may be reasonably doubted and all of it requires much careful discussions before we can be sure that we have stated it in a form that is wholly true." The present author considers that many of our concepts and axioms which are extensively been applied in earth sciences for a long time as authentic and of paramount importance, require sensible evaluation, and, if necessary, modification. The author would be satisfied if he can utilize the rich scientific heritage developed through protracted studies by the scientists from all over the globe in an honest and meaningful manner avoiding fairy tale-like imagination and dogma. Since the view of constant dimension earth throughout the past geological time has been rejected as per the reasoning earlier mentioned, the concept of plate tectonics would also be automatically discarded. However, plate tectonics [7] happen to be one of the most popular views - or, perhaps the most remarkable concept ever developed in earth science that enjoys support from a large section of geo-scientists. Hence, for rejecting such a popular view it is necessary to discuss the drawbacks 


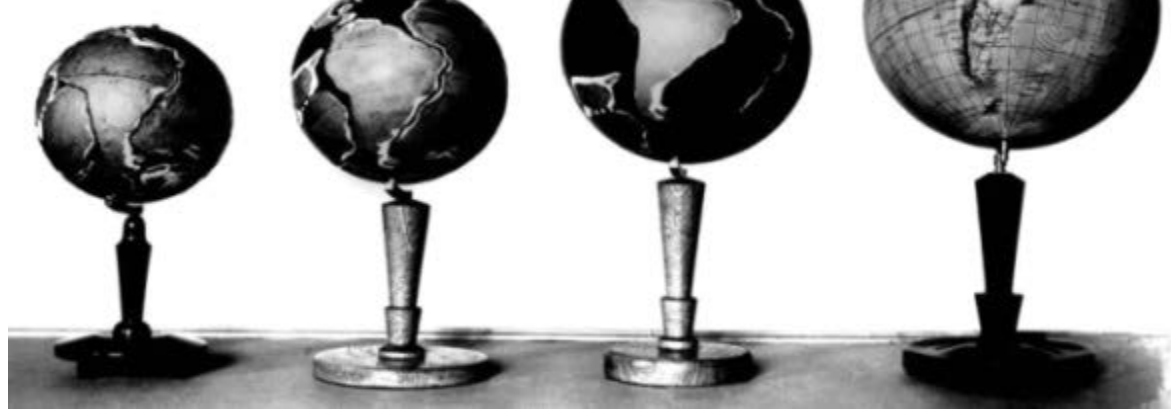

Figure 1: O. C. Hilgenberg of Germany in 1933 showed that if the radius in a model of Earth could be reduced to two-third of its radial thickness, all the continental blocks could be adjusted in a perfect manner. It can be noted that in the primordial small Earth, there were no oceans although lakes were present. Hence, it can be conceived that at that time the ocean-forming water was associated with the Mantle turning it considerably fluid and predominantly suitable for expansion (Sen, 1984-2007).

of the concept. Essentially plate tectonics is based on the concept of uniform earth dimension or unchanged dimension of the globe throughout the past geological ages and requires several fabricated assumptions for explaining only a few features of the earth system, restricted mainly to the crust of the planet. The concept does not convey any idea regarding the origin of continents or oceans, neither it gives information on interior of the planet. To keep the dimension of the planet constant it views that while a plate or solid continental fragment plunges into another solid part of the earth in a smooth conveyer belt like manner, in another part of the planet new plates are emerged, defying basic principles of physics. The process is operated owing to a convection current that occurs in the mantle which is solid and rigid as confirmed by propagation of seismic waves. The movement, though occurs in extremely slow manner, is capable of drifting the continents to great distances, concurrently driving a geodynamo, responsible for magnetic features of the planet.

\section{Unified Global Tectonics}

The concept of unified global tectonics (Sen, 1984, 1986, 2003, 2007) [8-11] has been developed based on the earth expansion theory of Hilgenberg (1933) [5] which envisages that if we could reduce the radius of the earth to two-third of its present thickness, all the continental blocks would be perfectly adjusted in the resultant small globe. Such small globe would be devoid of ocean basins although a few epicotinental seas or lakes were present. Therefore, it can be deduced that the ocean-forming water at that stage must have been associated with the mantle. Under such a set up, the original mantle material would be considerably fluid and consequently highly suitable for the manifestation of expansion, leading to continental fragmentation and various related features. The view is based on the results of experimental studies conducted by Roy and Tuttle (1961) [12] confirming depression of melting point of silicate rocks under hydrothermal and high pressure condition. As evidenced by the tidal pull of Moon, it is rational to conclude that the reason of the earth's expansion is the gravitational pull exerted by the Moon causing periodical bulging of the semi-fluid mantle. However, with progress of the process due to escape of volatiles from the mantle consisting chiefly of water, the ocean basins would be filled up with water simultaneously reducing the fluidity of the mantle which would eventually turn into a rigid geosphere. As explained above, the Moon - responsible for causing earth's expansion by exerting tidal pull - was originally an independent small planet which was captured by the earth when it was approaching the latter. Initially due to the Moon's magnetic influence exerted over the magnetic core of the earth, the latter's spatial orientation was drastically changed, causing major alteration in earth's climatic features. It has been conceived that originally in the small earth the solid iron core and mantle - initially which was adequately fluid - were juxtaposed to each other. As has been discussed, an appropriate gravitational force from an extra-terrestrial planetary body - the Moon - was responsible for the earth's expansion by bulging up of the semi-fluid mantle causing rupture of the original core-mantle conjunction. Also, due to such bulging of the semi-fluid mantle a number of expansion cracks - that eventually turned into mid-oceanic ridges - were produced over the solid sialic crust through which large quantum of basic magma disgorged and spread on both sides of the cracks to form ocean basins. Matching thickness of the fluid outer core with that of the extent of expansion, strongly supports the view that in consequence of prolonged expansion, along the ruptured core-mantle conjunction a void zone, identified as outer core in the prevalent concept, was gradually opened up. In due course the void zone would turn into a pseudo-fluid or virtually void geosphere owing to influx of fine particles from the adjoining mantle. The above mentioned arguments lead us to envision that two rocky and metallic geospheres separated by a non-solid and virtually void zone occur in the deep interior of the planet (Figure 2). This sort of internal setup would give rise to the phenomenon of reverse gravity generating low temperature and low pressure zones in the earth's deep interior. This simple and down to earth concept explained here is in complete agreement with all the major fundamental features, including several hitherto unexplained ones encountered in the planetary interior. Evidences of low temperature and low pressure zones in the planet's deep interior are exhibited in meteorites which are considered to be broken fragments of a pre-existing planet. These evidences include strong magnetic nature of some meteorites, texture of iron meteorites, presence of minerals with hydrocarbons, amino 


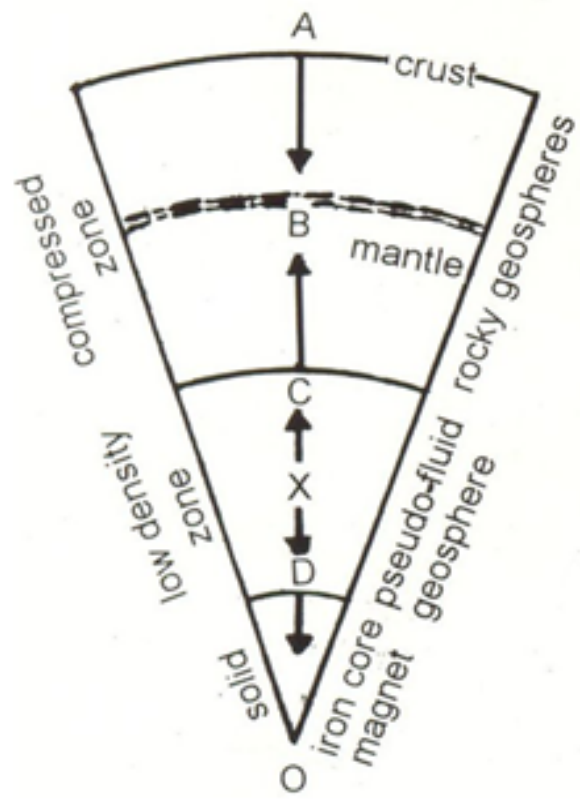

Figure 2: Overall structure of the earth (not to scale) showing trend of gravitational attraction on surface and interior of the planet. At the centre of the planet the trend of gravitational attraction would be oppositely directed. With separation of original semifluid mantle from the iron core due to planetary expansion and formation of a virtually void zone in between - the so called outer core of the prevalent view -, it is reasonable to conceive that around the inner core oppositely or reversely directed force of gravitational attraction would prevail forming a low pressure low temperature zone at depth.

acid and water in some rocky meteorites, as well as, globular shape of chondrules - all of which unmistakably confirm low temperature and low pressure condition in the deep interior of the planet. With the new concept the picture that emerges on the interior of the earth is this that during the Precambrian era, when the planet's core and mantle were juxtaposed to each other, extra-terrestrial magnetic influence caused movement of the iron-core, as a result of which the planet as a whole was tilted or deflected in space. Because of such alteration in spatial orientation of the planet, remarkable changes in the geographical features in various parts of the globe occurred, signatures of which, like the polar region revamping in to a hot zone and vice-versa, are documented over the global surface (Figure $3 b)$. In contrast, when the pseudo-fluid or void geosphere attained adequate thickness due to prolonged expansion, (Figure 3d), instead of deflection of the entire earth, only its magnetic iron core started to execute smooth and secular movements, including reversal of the poles, which are precisely documented over the surface rocks (Figure 3). It is understandable that in case of expansion, the sialic super-continent would be fragmented because of development of a number of expansion cracks, followed by widespread expulsion of basaltic magma through the cracks that would occupy the new space generated along the cracks forming rudimentary ocean basins. Under such circumstances instead of collision, the fragmented parts would tend to move away from one another causing enhancement of the ocean basins. Geological records, on the other hand, confirm collisions of continental plates have actually occurred at certain places during the past geological periods. In unified global tectonics this has been attributed due to the force generated by rotation of the planet along its axis of rotation which would be maximum around the equator and minimum near the poles. This sort of force generated

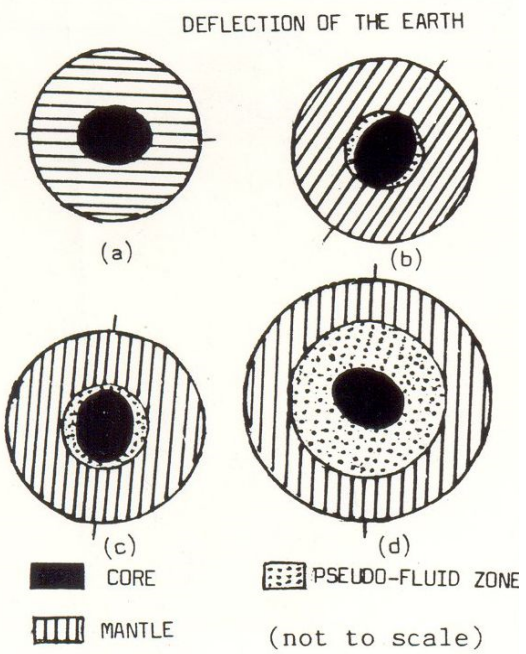

Figure 3: Before expansion of the planet, the solid iron core and semi-fluid mantle were juxtaposed to each other (Figure 3a). Due to expansion the original core-mantle conjunction was ruptured (Figure $3 \mathrm{~b}$ ) and along the ruptured surface a void zone was developed which gradually grew into a pseudo-fluid geosphere, i.e., the earth's fluid outer core (Figure 3c). This view supports that two geospheres of rocky and metallic composition, separated by a non-solid and virtually void zone, occur in the deep interior of the planet. Such disposition of the planet would give rise the phenomenon of reverse gravity in the planet's deep interior and core generating low temperature and low pressure zones in the earth's core and deeper parts. After development of the broad pseudo-fluid or virtually void geosphere (Figure $3 \mathrm{~d}$ ), the iron-core would remain within it in a suspended condition. Hence at this stage, pertaining to younger geological periods, in response to extra-terrestrial magnetic influences, new geomagnetic phenomena, like, polar wandering, pole reversal and west-ward drift could take place.

due to rotation or spin of the planet was not only responsible for continental collision forming colossus structures like the Himalayas but also caused major revamping of the continental fragments and ocean basins.

\section{Remarks and Conclusions}

The author in the book "Earth - Planet Extraordinary" has advanced the following new concepts:

1. The globe in its present outward appearance shows occurrence of continents in disjointed form which cannot be adjusted in appropriate manner whereas in a smaller globe, reduced to two-third of its present radial dimension (Hilgenberg, 1933), proper fitting of the continents can be achieved. This unmistakably demonstrates that the earth is an expanded planet, though in case of solid and rigid condition of the mantle planetary expansion and continental drift would not be possible.

2. The author has pointed out that since the small earth of unexpanded stage was devoid of oceans, the mantle at that stage must have been sufficiently fluid or semi-fluid owing to incorporation of ocean-forming water - an essential requirement for earth's expansion or continental drift. The view has been developed based on the work of Roy and Tuttle (1961) [12] who showed that melting point of a silicate rock under hydrothermal and high pressure condition would be depressed or, in other words, it would develop considerable fluid characteristic. 
3. It has been postulated that a planetary body came near the earth and was eventually captured by the latter, thereby turning the former a solitary satellite of the earth, the Moon. Since then the Moon started to rotate around the earth and due to its gravitational attraction on the earth, chiefly affecting the semi-fluid mantle, caused its expansion. As the Moon was revolving around the earth and the Moon too was rotating around its own axis of rotation, the earth's expansion was manifested in a uniform manner.

4. It was owing to expansion earth's solid sialic crustal cover developed a number of long sinuous cracks along which the planet's super-continent was fragmented forming several units of continents.

\section{References}

1. Ortelius Abraham (1570) Thesaurus Geographicus (in Latin) 3rd Edition, Antwerp Plantin. OCLC 214324616.

2. Snider-Pellegrini Antonio (1858) La Creation et ses Mysters Devoiles, Librarie A. Franck \& Dentu, Paris.

3. Wegener Alfred (1912) Die Entstehung der kontinente, Petermann Mitteilungn, 185195,253-256,305-309.

4. Yarkovsky, Ivan Osipovich (1888) Hypothese cinetique de la Gravitation universelle et connexion avec la formation des elements chimiques, 134p, Moskau.

5. Hilgenberg OC (1933) Vom Wachsenden Erdball, Berlin: Giessmann \& Bartsch.

6. Russell, Bertrand (1912) The Problems of Philosophy, Home University Library, Oxford University Press paperback, 1959 Reprinted, 1971-1972.

7. Dietz Robert S (1977) Plate tectonics: A revolution in geology and geophysics. Tectonophysics. 38: 1-6.

8. Sen Subhasis (1984) Unified global tectonics - a new qualitative approach in Earth sciences, Indian Science Congress, Geology and Geography Section, Ranchi, January 3-8, 71st Session, Abstract, p.23-24,.Also in Jour. Min. Met. \& Fuels 32: 20-22.

9. Sen Subhasis (1986) A new concept on the Earth's interior, geomagnetism and crustal fragmentation, International Symp. on Neotectonics in South Asia, Survey of India, Dehra Dun, Feb.18-21, Proceedings, p.83-94.

10. Sen Subhasis (2003) Unified global tectonics: structure and dynamics of the total Earth system, Indian Science Congress, Earth System Sciences, Bangalore, January 3-8, 2003, 90th Session, Abstract, p.57-58,. Also in Jour. Min. Met. \& Fuels 51: 351355 .

11. Sen Subhasis (2007) Earth - The Planet Extraordinary, Allied Publishers Ltd., New Delhi, 232.

12. Roy R, Tuttle OF (1961) Investigation under hydrothermal conditions, In Ahrens, I.H., Rankama, A.K. and Runcorn, SK (eds.) Physics and Chemistry of the Earth 1: 138-180 Pergamon Press, New York.

\section{Citation:}

Sen S (2021) Earth Science Needs Thorough Introspective Analysis and Renovation. Geol Earth Mar Sci Volume 3(3): 1-4. 